\title{
Systems Biology - Herausforderung und Anregung für die Komplementärmedizin
}

\author{
Herbert Schwabl, Cécile Vennos \\ Padma AG, Research Department, Schwerzenbach, Schweiz
}

\begin{abstract}
obald wissenschaftliche Revolutionen von Politikern angekündigt werden, ist Vorsicht geboten. Dies war schon 1971 der Fall, als Präsident R. NIXON in seiner Rede an die Nation den „war on cancer“ ausrief und die ersten 100 Millionen US\$ für die Suche nach einem Krebsheilmittel zur Verfügung stellte [1].
\end{abstract}

\section{Die Entschlüsselung des menschlichen Genoms}

Fast dreissig Jahre und viele weitere 100 Milliarden Forschungsgelder später war es wiederum so weit, als Präsident B. Clinton und Premier T. BlaIR am 26. Juni 2000 die vollständige Kartierung des menschlichen Genoms verkündeten [2]. Die Wissenschaft folgte ein halbes Jahr später mit den Schlüsselpublikationen in Nature am 15. Februar [3] und Science am 16. Februar 2001 [4]. In der Folge wurden die Genome einer ganzen Reihe von Pflanzen, Tieren und Mikroorganismen decodiert. Die erfolgreiche Gensequenzierung zusammen mit der Archivierung und Auswertung in leistungsfähigen Computersystemen führte zu einer fast unüberschaubaren Datenflut, zusammen mit neuen Begriffen und der Entstehung neuer Wissenszweige [5].

\section{Erste Widersprüche}

Gleichzeitig mit den genannten Schlüsselpublikationen in Science und Nature erschien in Lancet ein Editorial, das die damalige Euphorie bereits relativierte und auch erste Widersprüche an-

Die Systembiologie wird als neue wissenschaftliche Disziplin mit grossem Aufwand in der Schweizer Forschungslandschaft etabliert. Sie versteht sich als Zusammenspiel der einzelnen Wissensdisziplinen, die sich aus der Genetik entwickelt haben, der so genannten Omics: Genomik, Proteomik und Metabolomik. Der vorliegende Beitrag diskutiert die Möglichkeiten und Grenzen dieses Forschungsansatzes und die neuen Herausforderungen, die die Systembiologie an die Komplementärmedizin stellt.

Schlüsselwörter: Systembiologie, Komplementärmedizin, CAM, Genom

\section{Systems Biology - \\ Challenges and Suggestions for Complementary Medicine}

Systems biology is a novel scientific discipline which is currently being advanced in the Swiss scientific landscape with immense financial backing. It is characterized by proponents as the interaction of the different scientific fields that have developed from genetics, the so called 'omics': genomics, proteomics, and metabolomics. Possibilities and limits of this research approach are discussed as well as new challenges posed to complementary medicine by systems biology.

Key words: Systems biology, complementary medicine, CAM, genome

sprach [6]. Die ursprünglichen Berechnungen gingen davon aus, dass das menschliche Genom aus etwa 100'000 Genen besteht. Bei der genaueren Vermessung musste diese Schätzung auf nur etwa 20'000 Gene reduziert werden. Das war deshalb besonders verwunderlich, weil sich auch einige sogenannt niedere Tiere, wie etwa der Fadenwurm, in derselben Grössenordnung finden. Offensichtlich korreliert die Komplexität der Organismen nicht mit der Anzahl ihrer Gene.

Der damals umstrittene Forscher und Unternehmer GREG VENTER berichtete ausserdem, dass zwei Individuen mehr als 99.9\% identische Gensequenzen aufweisen, was er als einen Angriff auf das „gloriose“ Konzept des Individuums interpretierte [4].
Obwohl diese Erkenntnisse bereits erste Fragen aufwarfen, glaubten die Gen-Mediziner, nun das Werkzeug in Händen zu halten, um die Krankheiten zu verstehen und endlich die offenen Probleme der Medizin zu lösen. Die Zeit war geprägt von einer euphorischen Stimmung für die Gentechnik in den Bereichen der Medizin und der Biowissenschaften. Forschungsprogramme wurden eingerichtet mit der Überzeugung, nun die Krankheiten in den Griff zu bekommen.

Als eine Art Zwischenbilanz kann man aber heute feststellen: Die grossen Erfolge blieben aus und es kam z.B. nicht zu dem erwarteten Zuwachs an Neuzulassungen von pharmazeutischen Wirkstoffen [7], denn die Rate der Neuzulassungen blieb über Dekaden nahezu gleich, siehe Abb.1. 


\section{Das Versagen des mono- genetischen Ansatzes}

Die aus der Molekularbiologie übernommenen Ansätze basierten anfangs auf der Theorie der deterministischen Genetik, die von einem direkten Weg vom Gen zum Protein zur Funktionsgruppe ausgeht. Bildlich dargestellt ist das Genom die Abfolge der vier Nukleinsäuren auf dem DNA-Strang, sozusagen ein Text aus den Buchstaben A, C, $\mathrm{T}$ und G. Durch Ablesen von Genomsequenzen werden die Proteine gebildet, so wie aus Buchstaben Worte [8]. Aufbauend auf die Genomik wurde auch erforscht, wie die Proteine weiter in funktionellen Einheiten, in Organellen und Zellen zusammenwirken. Parallel dazu entstanden neue Fachrichtungen: Proteomics, die sich mit der Gesamtheit der in einer Zelle oder einem Lebewesen bei bestimmten Bedingungen vorhandenen Proteine befasst, und Metabolomics, welche sich mit dem gesamten Stoffwechsel und den Interaktionen zwischen verschiedenen Stoffwechselwegen auseinander setzt. Im Jargon wird „Omics“ als kurzer Sammelbegriff für diese neuen Fachrichtungen verwendet [9].

Aus dem deterministischen Ansatz blieb die Überleitung zu komplexen, multimodalen Krankheiten, wie etwa der Arteriosklerose, schwierig. In einer ersten Analyse nach der Entschlüsselung des menschlichen Genomsatzes war schnell klar, dass von den entdeckten Genen etwas weniger als 1000 als spezifische „Krankheitsgene“ (disease genes) in Frage kommen [10]. Die meisten davon sind für seltene monogenetisch bedingte Krankheiten verantwortlich. Ausserdem war ersichtlich, dass eine grosse Vielfalt von möglichen Gensequenzen und daraus abgeleiteten Proteinen im Krebsgeschehen eine Rolle spielen können und dass das typische „Krebsgen“ nicht existiert. Vielmehr sind alle Gene mögliche Kandidaten für die Entstehung von Tumorerkrankungen [11]. In verschiedenen Umgebungen und unter Einfluss verschiedener Randbedingungen sind bei derselben Genausstattung unterschiedliche Ausgestaltungen von Organismen möglich bis hin zu ihren (Erb-) Krankheiten und Krankheitsprädis-

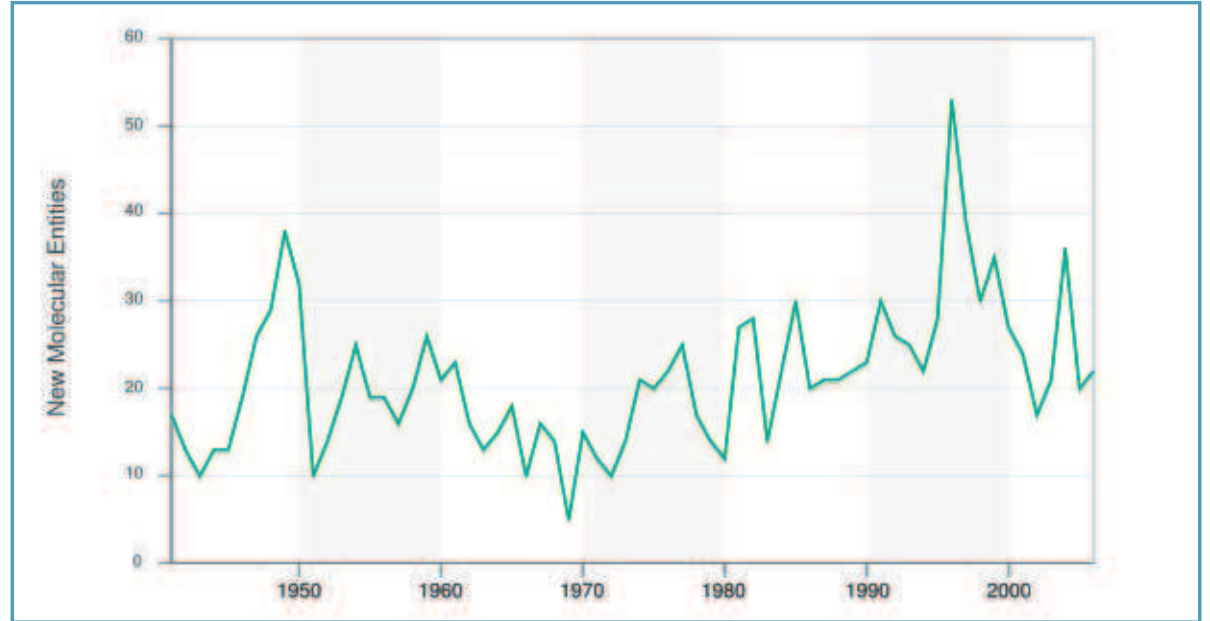

Abb. 1. Zulassung neuer molekularer Wirkstoffe durch die FDA [7]. Seit den Anfängen der Arzneimittelzulassung bewegt sich die jährliche Zahl zugelassener Wirkstoffe bei etwa 10-20. Erst ab 1970 bewegt sich der Trend langsam nach oben und liegt heute zwischen 20-30 neuen Wirkstoffen pro Jahr. Die kurze Spitze um 1997 wurde durch eine administrative Änderung der Zulassungsbedingungen bewirkt.

positionen. Verschiedenen phänotypischen Ausprägungen kann derselbe Genotyp zu Grunde liegen, denn es gibt keine Eins-zu-eins-Entsprechung von Gen / Gendefekt zu Phänotyp. Mit der vollständigen Entschlüsselung des menschlichen Genoms zeigte sich bereits 2001, dass die deterministische, monogenetische These höchstens in ganz seltenen Fällen zutreffen kann, und es war schon damals ersichtlich, dass die Gentherapie nur in wenigen Bereichen Erfolge bringen kann.

Dazu nochmals Greg Venter: ,There are two fallacies to be avoided: determinism, the idea that all characteristics of the person are 'hard-wired' by the genome; and reductionism, the view that with complete knowledge of the human genome sequence, it is only a matter of time before our understanding of gene functions and interactions will provide a complete causal description of human variability. The real challenge of human biology, beyond the task of finding out how genes orchestrate the construction and maintenance of the miraculous mechanism of our bodies, will lie ahead as we seek to explain how our minds have come to organize thoughts sufficiently well to investigate our own existence“ [4].

\section{Von den Omics zur Systembiologie}

Um diese Kurzsichtigkeit des deterministischen Ansatzes zu beheben, wurde einige Jahre später das Schlagwort der Systembiologie (engl: systems biology) populär. Ziel dieses Wissenschaftszweigs ist es, mit den entschlüsselten DNA-Sequenzen (Buchstaben) und Genen (Worte) ganze Sätze und Kapitel zu schreiben [8]. Erst das syntaktische „In-Sätze-Fassen“ erlaubt das Verständnis des dynamischen Netzwerks der ineinandergreifenden biologischen Elemente. Diese umfassen die biochemischen und zellulären Abläufe bis hin zum Gesamtorganismus und finden nicht nur auf einer Gen-zu-Gen-Basis statt [12]. In der Systembiologie wird die in den Einzeldisziplinen gewonnene biologische Information zusammengeführt und somit die zersplitterten Forschungsrichtungen der Omics zu einem Gesamtverständnis integriert [8].

Die Systembiologie im Wechselspiel von Theorie, Beobachtung und Experiment soll damit der Biologie helfen, von einer qualitativen zu einer quantitativen Wissenschaft zu werden, analog der Chemie und Physik. Die Wissenschafter werden damit zu Wissenschaftern/Ingenieuren [13]. Diese Hinwendung könnte einerseits insofern als vielversprechend gedeutet werden, da sich gerade in den harten Wissenschaf- 
ten, wie etwa der Physik, in den letzten Jahren eine Bewegung weg vom Reduktionismus hin zur Emergenzforschung abzeichnet [14]. Es bleibt abzuwarten, ob sich die Systembiologie dieser Strömung anschliesst und sich an der Erforschung emergenter Systeme beteiligt, oder ob sie zu ihrem Beginn - wie die angeführten Leitwissenschaften Chemie und Physik - eine jahrelange Phase des reduktionistischen Determinismus durchlaufen muss.

Die Vision der verschiedenen Omics, und damit auch der Systembiologie, für die Entwicklung der Medizin ist es, dass die Verfügbarkeit von immer mehr individuellen Daten eine optimierte medizinische Betreuung des Einzelnen ermöglicht. Die persönlichen Daten werden dann mit den bestehenden Datenbanken korreliert. Softwaregestützte, probabilistisch aufgebaute Entscheidungen werden vorstellbar, die dazu führen, die diagnostischen, präventiven und therapeutischen Interventionen $\mathrm{zu}$ überprüfen bzw. zu planen [13].

Dazu wird die Variabilität zwischen den Individuen in einzelnen Nukleotiden des DNA-Stranges (Single Nucleotide Polymorphism - SNP) erhoben und die Varianten werden gesammelt und in Datenbanken abgelegt [15]. Gleichzeitig wird die Wirkung von pharmazeutischen Wirkstoffen auf die verschiedenen Gensequenzausstattungen bzw. deren Träger studiert. Das führt dazu, dass auf den individuellen Patienten zugeschnittene Therapieangebote zur Verfügung gestellt werden können. Voraussetzung dafür ist die Analyse des individuellen Genoms, Klassierung anhand der SNP und Abstimmung allfälliger pharmazeutischer Interventionen durch die Pharmakogenetik [16]. Diese „personalisierte Medizin“ wird so neu definiert und es können genetisch massgeschneiderte Therapieformen angeboten werden. Damit wird die personalisierte Therapie, die bis anhin eine der Domänen der Komplementärmedizin war, in ein neues Umfeld geführt. Die Komplementärmedizin wird also vermehrte Anstrengungen unternehmen müssen, um sich auf diesem Gebiet weiter behaupten zu können.

\section{Die Schweizer Anstrengung}

Um in der internationalen Forschung nicht ins Abseits zu driften, wurde letztes Jahr in der Schweiz ein Rettungsanker ausgeworfen: Im ProjektX.ch fördert die Schweiz mit beispielhaftem Einsatz das neue Gebiet der Systembiologie. Innerhalb der nächsten 4 Jahre sollen 400 Mio. CHF in dieses Projekt fliessen, bezogen auf die Bevölkerungszahl bislang der weltweit höchste Förderbetrag zu diesem Thema, gleichzeitig auch die grösste einzelne Forschungsanstrengung in der Geschichte der Eidgenossenschaft [17].

Explizit soll diese Forschungsanstrengung die Pharmaindustrie aus ihrer Innovationskrise herausholen, die nicht zuletzt dem monogenetischen Determinismus $\mathrm{zu}$ verdanken ist. In Bezug auf Krankheiten soll die Systembiologie helfen, die Ambiguitäten der medizinischen Therapie aufzulösen und den klinischen Entscheidungsprozess weg von der Empirie zu einem gänzlich mechanistischen Ablauf zu führen [12].

\section{Vorsicht und Kritik}

Hier ist vielleicht der Punkt, davor zu warnen, dass Ingenieure sich mit ihrem Denken verselbstständigen und objekthafte „Maschinen“ als oberstes Ziel und unabhängig von ihrer Umwelt sehen, dass Wissenschaftler der „Maschinerie“ Entscheidungen überantworten und sich deshalb ihrer persönlichen Verantwortung enthoben glauben. Der „Maschinerie“ - hier kann es sich z.B. um Apparate, Konzepte wie Hierarchien, den Zufall oder eben die Gene handeln - wird dabei eine absolute Objektivität zugesprochen, obwohl man ihre Funktionsmechanismen und ihre Auswirkung auf die Umwelt nicht genau genug versteht. Gemäss NoRBERT WiENER, dem Schöpfer der Kybernetik und damit auch einer der Vorväter der Systembiologie, entarten derartige Wissenschafter/Ingenieure zu so genannten „gadget worshipers“ [18].

Die Kritik an der Systembiologie mag vielleicht überzogen oder strukturkonservativ wirken, es muss jedoch bedacht werden, dass manche Forscher sie nicht nur beobachtend experimentierend verstehen, sondern auch als schöpferisch sehen; nicht nur auf abstraktes mathematisches Verständnis abzielend, sondern auch die Erwartung schürend, im Rahmen der „synthetischen Biologie“ neue und verbesserte biologische Funktionen zu schaffen.

Durch geschickte Kombinationen gut charakterisierter biologischer Einzelteile wird das Erschaffen neuartiger synthetischer Ganzheiten möglich [13]. Auf diese Weise neugeschaffenes Leben, in salopper Formulierung „Life version 2.0“ genannt, soll neue Funktionalitäten und bessere Leistungsfähigkeit bringen. Im Rahmen der „predictive biology“ besteht auch der Anspruch, neuartige, biologische Prozesse mit bestehenden Komponenten vorherzusagen [12]. Der Ingenieur wirkt hier als Demiurg, quasi als Schöpfergott, der selbst eine neue Welt erschafft. Nochmals sei hier N. WIENER zitiert, der schon zu Beginn der Systemtheorie gefragt hat: „Can God play a significant game with his own creature?" Hat also das neu erschaffene Wesen das Potential, den Demiurgen zu überraschen? Wir wissen, dass Lebewesen dieses Potential in sich tragen. Wiener ahnte bereits, dass dies auch eine Frage für die Wissenschafter ist, denn er transformierte diese Frage zu „Can any creator, even a limited one, play a significant game with his own creature?" [18] Eine schwierig zu beantwortende Frage, übrigens auch im Umkehrschluss: Kann der Erschaffende die in die Welt geworfene Kreatur genügend kontrollieren? Mit diesen Fragen muss sich nicht nur die Systembiologie, sondern in letzter Konsequenz die globale Weltgesellschaft auseinandersetzen.

Vielleicht gründet das Vertrauen der Systembiologen in die kommenden Möglichkeiten auf der bislang unspektakulär verlaufenen, natürlichen Phylogenese. In natürlichen biologischen Systemen konnte man sich auf eine grosse Einschränkung der möglichen, d.h. lebens- und propagierungsfähigen GenVariationen durch den evolutionären Druck auf die biologischen Systeme und Subsysteme verlassen. Diese wurden durch die evolutionären Mechanismen bereits optimal an die Aufga- 
ben angepasst (time-tested) und sind damit auch robust gegenüber Umwelteinflüssen. Nur in Ausnahmefällen und bei Vorhandensein geeigneter Vektoren (im Sinne von Pandemien) konnte sich das biologische Gefährdungspotential manifestieren. Soweit auch eines der Hauptargumente für die Ungefährlichkeit klassischer Genfreisetzungsversuche. Bei mechanischen Maschinen gilt gemeinhin die Annahme, dass wir vor der Inbetriebsetzung die Auswirkungen genau abschätzen können. In Kenntnis der verschiedenen Risken, denen uns das globale Ensemble der technischen Maschinen bislang ausgesetzt hat, ist zu bezweifeln, ob dann die viel komplizierteren, nichtlinearen, verkoppelten biologischen Regelkreise den zielgerichteten Vorgaben der Ingenieure folgen werden.

Aber natürlich darf man auch die Resonanz in der Gesellschaft nicht unberücksichtigt lassen. In Erinnerung bleibt in der Schweiz die GentechfreiInitiative, die 2005 von $55,7 \%$ der Stimmenden angenommen wurde und $\mathrm{zu}$ einer Beschränkung der Genmanipulation an Nutzpflanzen und Nutztieren führte [19]. Damals wurde die medizinische Forschung von diesem Moratorium noch ausgeklammert. Insofern ist zu erwarten, dass im helvetischen ProjektX.ch die Sache vorsichtiger angegangen wird.

\section{Herausforderungen, Anregungen}

Die Komplementärmedizin ist bezüglich des durch das Gen-Paradigma induzierten Erkenntnisgewinns bisher äusserst zurückhaltend geblieben. Dies kann einerseits z.T. auf die Ängste der Bevölkerung, Angst vor Experten, die Anti-Gentech-Bewegung, Kritik an Stammzellforschung, Klonen, etc. zurückgeführt werden. Die Komplementärmedizin ist ja auch mit dem alternativen Gedankengut verbunden und damit auch Teil dieser kritischen Bewegung. Weitere Gründe für diese Zurückhaltung mag auch an mangelnden finanziellen Ressourcen liegen und an dem beträchtlichen Zeitaufwand, der nötig wäre um sich in das Thema $\mathrm{zu}$ vertiefen.
Dabei ist gerade ein integratives Denken den verschiedenen Vertretern der komplementärmedizinischen Disziplinen eigen und es ist ihnen z.T. erstaunlich gut gelungen, mit solchen integrativen Ansätzen altes und neues Wissen miteinander zu verbinden. Dennoch darf nicht übersehen werden, dass durch die enormen globalen Forschungsbemühungen auch enorme Mengen an neuen Erkenntnissen gewonnen werden, die nach und nach in den Alltag einströmen werden. So bringt jeder Jahrgang frisch promovierter Mediziner und Wissenschafter diese neuen Begriffe in die Gesellschaft und somit auch in die Komplementärmedizin.

Die Systembiologie bringt einen rasenden Fortschritt. Neben den klassischen Forschungstugenden Beobachten, Prüfen und Planen kommen diejenigen des Internetzeitalters dazu: $\mathrm{Zu}$ sammenfassen und Teilen (merge and share). Die Technologie um das Wissen aus verschiedenen Forschungszweigen auch über grosse Distanzen zu nutzen ist heute selbstverständlich. Es geht aber weiter: Wissensbausteine, die aus Studien von Hefekulturen, Würmern oder Fliegen gewonnen wurden, werden mit dem Wissen um das menschliche Genom verschmolzen. Die Systembiologie soll diese Prozesse durch die Integration weiterer Wissenszweige noch beschleunigen [13].

Bei aller vorher geäusserten Kritik: Ist dies nicht der Idealzustand eines strebenden Forschers, sogar von Humboldt'scher Prägung: Einbeziehung der verschiedenen Wissenschaften, interdisziplinäre Zusammenarbeit, Teilen und Mitteilen des Wissens in einer globalen Forschergemeinschaft?

Um diese erweiterten Möglichkeiten pharmakologisch zu nutzen, ist der Aufbau einer riesigen Datenmenge nötig, weshalb sich dies für den einzelnen Patienten erst nach und nach in einer nutzbaren Änderung von Behandlungsstrategien niederschlagen wird. Die Frage stellt sich, wie die Zulassungsbehörden auf die Tatsache reagieren werden, dass viel mehr Informationen über Wirkungen und Risiken von Pharmazeutika in verschiedenen Patientengruppen gewonnen werden können. Dazu Jones: „... the FDA will respond to new knowledge as it emerges, updating labels as necessary”. Er zitiert zu diesem Thema auch LAWRENCE LESKO, Direktor der FDA's Office of Clinical Pharmacology, Center for Drug Evaluation and Research: „You don't wait until you have all the information for every genotype at every dose level for every combination of chemotherapy before you label a drug product. I think that would be unwise and probably inconsistent with the regulatory mandate" [16]. Es ist abzusehen, dass sich die regulatorischen Hürden auf Grund der Kenntnisse auf dem Gebiet der Genwissenschaften erhöhen werden: Neue Erkenntnisse werden auch von Seiten der Arzneimittelbehörden zu neuen Anforderungen für die Arzneimittelzulassung führen. Für den traditionellen Arzneimittelschatz der Komplementärmedizin ist hier zu fordern, dass diese zukünftigen Entwicklungen nicht die Zulassungsbedingungen für die bewährten Heilmittel der Komplementärmedizin weiter erhöhen. Hier ist vorsichtiges Beobachten angezeigt.

Eine weitere Herausforderung besteht für die traditionellen Medizinsysteme. Ein auf prä-naturwissenschaftlichem, empirischem Fundament ruhendes Theoriegebäude tut sich klarerweise schwer, Aspekte der neuzeitlichen Wissenschaft zu integrieren. Die Kenntnisse der diskreten Naturbausteine wie Atome, Moleküle und Zellen, also auch die der Gene, sind diesen Systemen an sich fremd. Dies gilt übrigens auch für die komplementärmedizinischen Systeme, die seit Paracelsus in Europa als Gegenentwurf zur etablierten Wissenschaft formuliert wurden (wie etwa Homöopathie, Spagyrik und Anthroposophie).

\section{Das Beispiel Traditionelle Chinesische Medizin TCM}

Auch in China ist der heutige Zugang zur TCM analytisch-reduktionistisch: aktive Wirkstoffe isolieren und einzeln prüfen. Jedoch sind viele Arzneimittel der TCM, wie in anderen asiatischen Medizinsystemen auch, Vielkomponentengemische aus verschiedenen Arzneipflanzen; jede Formel enthält jeweils eine Unzahl von chemischen Mo- 
lekülen. Die Komplexität dieser Vielstoffgemische lässt sie für die Untersuchung mit den Werkzeugen der Systembiologie geradezu prädestiniert scheinen.

Tatsächlich gibt es im Rahmen der TCM mittlerweile Überlegungen, mittels Systembiologie die Reaktion des Gesamtorganismus auf die komplexen Präparate zu erfassen [20]. Indem viele Gene, Proteine und Metabolite gleichzeitig gemessen werden, kann aus deren Veränderungen auf die durch das Arzneimittel beeinflussten biochemischen Pfade rückgeschlossen werden [21].

Obwohl solche Untersuchungen bereits begonnen haben, könnte es noch etwas zu früh sein, um hier Erfolge vorzuweisen. Neben der Entwicklung besserer Detektionssysteme für die spezifischen Metaboliten sind erst noch die statistischen und computertechnischen Werkzeuge zu schaffen, um die enormen Datenmengen zu bewältigen. Zugleich läuft in China die Diskussion über Vor- und Nachteile einer Modernisierung der TCM und über die Art und Weise, wie diese aussehen kann und soll. Vielleicht muss die Technik der Systembiologie auch erst ihre Tauglichkeit in der Klinik und bei konventionellen Systemen zeigen, bevor sie bei so komplexen Themen wie Vielstoffgemischen aus asiatischen Medizinsystemen angewendet werden kann und einen lohnenswerten Beitrag zu deren Erforschung leisten kann.

\section{Integration und Modernisierung}

Für die Komplementärmedizin wird eine Auseinandersetzung mit diesen modernen Begrifflichkeiten der Systembiologie und der Omics unumgänglich sein. Ein konkreter Dialog mit Vertretern der neuen Wissenschaften, z.B: an Konferenzen, und eine Integration dieser Themen in die Ausbildung ist notwendig.

Übersetzungsarbeit ist ja für die Komplementärmedizin nichts Neues. Sie hat immer an dem sich weiter entwickelnden Rand der Medizin gearbeitet und häufig die Sprache anderer Kulturen und anderer Zeiten für eine praktische Umsetzung im modernen Umfeld aufbereitet. Die Systembiologie ist ein grosses Wissenschaftsgebiet, an das sich die Komplementärmedizin erst noch annähern muss.

Abschliessend noch ein Beispiel einer solchen Integrationsarbeit aus den Niederlanden, wo die Initiative 'Fusion' den wissenschaftsbasierten Dialog zwischen schul- und komplementärmedizinisch tätigen Ärzten fördert. Der Fusionkongress 2007 war den Themen der Systemtheorie gewidmet und zeigte auf, dass das Selbstverständnis der Medizin vom Disease Management weg hin zur Gesundheitsförderung geht. Dies begünstigt und benötigt zugleich integrative Ansätze, die moderne wissenschaftliche Erkenntnisse wie auch Methoden der konventionellen und der Komplementärmedizin vereinen. Die integrative Medizin beinhaltet so verschiedenste Themen, z.B. auch Systemische Medizin, Systembiologie, Immunologie, Phytotherapie und Asiatische Heilkunde [22]. Das Ziel mutet vertraut an: integrative Therapiekonzepte, bei denen der Patient, das Individuum, (wieder) im Zentrum steht.

\section{Literatur}

1 Nixon R 1971: http://dtp.nci.nih.gov/timeline/noflash/milestones/M4_Nixon.htm abgerufen am 20.Mai 2008

2 Clinton B 2000: http://www.genome.gov/ 10001356 abgerufen am 20. Mai 2008

3 The International Human Genome Mapping Consortium: A physical map of the human genome. Nature 2001;409:934-941.

4 Venter JC et al: The Sequence of the Human Genome. Science 2001;291:1304-1351.

5 Cook-Deegan R: Gene Wars: Science, Politics, and the Human Genome. W. W. Norton \& Company 1995.

6 Lancet 2001: Editorial: The human genome, in proportion. Lancet 2001;357:489.

7 FDA: http://www.fda.gov/oc/history/NDA approvals.html\#table abgerufen am 20.Mai 2008.

8 Aebersold R: Molecular Systems Biology: a new journal for a new biology? Molecular Systems Biology 2005; doi: 10.1038/msb 4100009

9 Omics: http://www.liebertpub.com/publication. aspx?pub_id=43 abgerufen am 20.Mai 2008

10 Jimenez-Sanchez G, Childs B, Valle D. Human disease genes. Nature 2001;409: 853-855.

11 Futreal PA, Kasprzyk A, Birney E, Mullikin JC, Wooster R, Stratton MR. Cancer and genomics. Nature 2001;409:850-852.

12 Liu E. Integrative biology and systems biology. Molecular Systems Biology 2005; doi: $10.1038 / \mathrm{msb} 4100008$

13 Church G. From systems biology to synthetic biology. Molecular Systems Biology 2005: doi:10.1038/msb4100007.
14 Laughlin R. A different universe. Basic Books, New York, 2005

15 SNP NCBI: http://www.ncbi.nlm.nih.gov/About /primer/snps.html abgerufen am 20.Mai 2008

16 Jones D. Steps on the road to personalized medicine. Nature Rev Drug Discovery 2007. 6:770-771.

17 Projekt SystemsX.ch: http://www.systemsX.ch and SystemsX.ch-Newsletter 14 Dec 2007.

18 Wiener N. God \& Golem, Inc. MIT press, Cambridge Massachusetts 1964.

19 Gentechfrei: http://www.gentechfrei.ch abgerufen am 20.Mai 2008

20 Wang $M$, Lamers RJ, Korthout HA, van Nesselrooij JH, Witkamp RF, van der Heijden $R$, Voshol PJ, Havekes LM, Verpoorte R, van der Greef J. Metabolomics in the context of systems biology: bridging traditional chinese medicine and molecular pharmacology. Phytother Res 2005;19:172-182.

21 Qiu J. A culture in the balance. Nature 2007; 448:126-128.

22 Bech F: Thinking out of the box. ECHAMP news, p4, Brussels November 2007. http://www.echamp.eu/fileadmin/user upload/News 2007/ECHAMP News Novem ber_2007.pdf abgerufen am 20.Mai 2008

\section{Disclosure Statement}

The authors declare that no financial or other conflict of interest exists in relation to the content of this article.

\section{Korrespondenzadresse:}

Dr. techn. Herbert Schwabl

Padma AG, Research Department

Wiesenstrasse 5, CH-8603 Schwerzenbach

h.schwabl@padma.ch 